\title{
Presencia de dimorfismo sexual entre patos Muscovy (Cairina moschata) en el traspatio de tres municipios de Guatemala
}

\section{Presence of sexual dimorphism between Muscovy ducks (Cairina moschata) in the backyard of three communities in Guatemala}

\begin{abstract}
Como citar el artículo
Jáuregui, R., et al. (2019). Presencia de dimorfismo sexual entre patos Muscovy (Cairina moschata) en el traspatio de tres municipios de Guatemala. Revista Naturaleza, Sociedad y Ambiente, 6 (1),29-38 DOI: https://doi.org/10.37533/ cunsurori.v6i1.39
\end{abstract}

Raúl Jáuregui, Mario E. Gonzáles, Carlos R. Lorenzo, Ana M. Folgar, Anna Isern. Peréz M. Parés-Casanova Instituto de Investigación, Centro Universitario de Oriente (CUNORI), Universidad de San Carlos de Guatemala

Recibido: 18 de julio de 2019 / Aceptado: 30 de septiembre de 2019

Disponible en internet el 29 de noviembre de 2019

*Autor para correspondencia, correo electrónico: rajauji57@gmail.com

\section{Resumen}

En este estudio, investigamos el dimorfismo sexual entre machos y hembras de patos Muscovy criados libremente en tres comunidades diferentes en Guatemala. El análisis se basó en 14 rasgos morfológicos importantes: longitud del ala, longitud del miembro posterior, alzada dorsal, longitud de la cabeza y ancho de la cabeza, diámetro longitudinal y bicostal, longitud dorsoesternal, altura de grupa, perímetros torácico y abdominal, perímetro del metatarso y peso corporal. Una muestra constituida por 137 machos y 225 hembras. Los machos presentaron una mayor masa que las hembras, pero eran menos robustos y patas. El análisis discriminante reveló que los machos no podían agruparse, pero las hembras de Camotán son distintas del resto. El análisis detallado demostró que la mayoría de las variables diferenciales (perímetros abdominales y del tórax) no estaban relacionadas con rasgos etnológicos, por lo que el grupo Camotán puede considerarse como un mero prototipo (asociado con la selección y reproducción artificial), no un ecotipo (como si lo que hace que los ecotipos sean adaptativos a las condiciones locales, este no es el caso). Los resultados de este estudio podrían ser efectivos en la caracterización y conservación de los recursos genéticos del pato Muscovy en Guatemala. Ahora es necesario estudiar la calidad de la canal y del huevo y otras variaciones a nivel molecular que aclararán aún más la similitud con los patos de otros orígenes para registrar esta raza internacionalmente.

Palabras clave: morfología, etnología, ecotipo, conservación

\begin{abstract}
In this study, we investigated the sexual dimorphism between males and females of Muscovy ducks freely bred in three different communities in Guatemala. The analysis was based on 14 important morphological features: wing length, rear limb length, dorsal elevation, head length and head width, longitudinal and bicostal diameter, eternal back length, rump height, chest perimeters and perimeter of the metatarsal and body weight. A sample consisting of 137 males and 225 females. Males had a higher mass than females but were less robust and legs. The discriminatory analysis revealed that males could not be grouped together, but Camotan's females are different from the rest. Detailed analysis showed that most of the differential variables (abdominal and chest perimeters) were not related to ethnological traits, so the Camotán group can be considered as a mere prototype (associated with selection and artificial reproduction), not an ecotype (as if what makes ecotypes adaptive to local conditions, this is not the case). The results of this study could be effective in characterizing and preserving the genetic resources of Muscovy Duck in Guatemala. It is now necessary to study the quality of the carcasses and egg and other variations at the molecular level that will further clarify the similarity with ducks of other origins to register this breed internationally.
\end{abstract}

Keywords: morphology, ethnology, ecotype, conservation 


\section{Introducción}

Una raza es un grupo homogéneo de ganado con rasgos físicos definibles, específicos e identificables (FAO, 2006) y sus individuos se distinguen por sus rasgos morfológicos y biométricos. El mejoramiento de los animales domésticos para satisfacer las necesidades humanas depende de la variación dentro y entre las razas, ya que dicha variación ofrece la oportunidad de selección.

La producción de patos es en gran medida una empresa tradicional y aún no se ha industrializado como la del pollo. A pesar de que el pato sigue siendo una especie olvidada entre las investigaciones sobre animales domésticos. Si el volumen de trabajo relacionado con la constitución fenotípica y genética de las razas criollas de Guatemala todavía es insipiente, la información sobre los patos domésticos criollos de Guatemala es nula.

El pato salvaje de Muscovy (Cairina moschata Linnaeus 1758) es originario de América (Donkin, 1989), su distribución comprende las costas de México, América Central y la mayoría de las regiones tropicales de América del Sur (Hernández, Muñoz, Valencia, Posso, \& Muñoz, 2017) (Schaaf, Gomez, Tallei, Rivera, Politi, \& Cuyckens, 2018). La especie salvaje, llamada Greater Wood Duck o Forest Duck, también tiene las patas proyectadas más hacia el frente con respecto a la base del cuerpo que los patos del género Anas, tiene garras fuertes y afiladas, dedos traseros bien desarrollados y alas anchas (Donkin, 1989), y un tamaño del macho promedio de 66 a 86.5 $\mathrm{cm}$, un peso corporal de 2 a $4 \mathrm{~kg}$, siendo el macho considerablemente más grande que la hembra (Donkin, 1989), (Baéza, Williams, Guémené, \& Duclos, 2001) (Almeida, 2014).
La especie doméstica derivada del pato salvaje de Muscovy se llama criollo, pato real, pato perulero, pato almizclado, pato mudo y pato Berbery. Es un animal rústico que no requiere instalaciones complicadas para su reproducción, es resistente a las enfermedades, tiene una alta prolificidad y presenta una buena precocidad en el engorde, así como una gran capacidad para aprovechar las raciones de alimentos (Igwe, Okoli \& Okeudo, 2003) (Almeida, 2014), (Abdeltawab, Salha, Allam, \& Fandy, 2017). Se crían principalmente para carne y huevos, aunque proporcionan otros materiales de valor económico como las plumas (Etuk, Abasiekong, Ojewola, \& Akomas, 2006).

En pequeñas comunidades de Guatemala, el pato Muscovy representa una fuente interesante de carne y huevo. La base de recursos alimenticios es la recolección, que consiste en desechos domésticos, cualquier cosa comestible que se encuentre en el entorno inmediato y pequeñas cantidades de suplementos de granos provistos por los lugareños. Los suministros de alimentos para el hogar y el medio ambiente varían según las actividades agrícolas en el hogar, la estación del año y el ciclo de vida de los insectos y otros invertebrados. Bajo este sistema actual, el pato Muscovy probablemente su productividad es mala y sigue siendo una especie de aves de corral perjudicada para la investigación.

El uso de diferentes medidas corporales disponibles ha llevado a varios investigadores a utilizar técnicas multivariadas para examinar simultáneamente la relación entre las medidas corporales y los rasgos de producción en pollo (Jáuregui, Flores, Vásquez \& Oliva, 2015), (Fitsum, 2015), pavo (Ogah, 2011), palomas (Uribe, Senar \& Camerino, 1985), (Parés-Casanova, 2013) y pato (Cuesta, 
2008), (Yakubu, 2011). Un primer paso para la investigación sobre el pato Muscovy en Guatemala es realizar una caracterización basada en los rasgos morfológicos fenotípicos (Oguntunji \& Ayorinde, 2014). Por lo tanto, se propuso el trabajo para estudiar los rasgos morfométricos de esta especie.

En resumen, los objetivos de este estudio fueron: examinar la morfometría del pato Muscovy de corral de Guatemala y el dimorfismo sexual; para determinar posibles ecotipos (entendida como una población adaptada localmente que se supone que es el resultado de la acción de la selección natural). Los resultados actuales podrían ayudar a mejorar las herramientas para la conservación y mejora de esta especie en el país.

\section{Materiales y métodos}

El estudio se ejecutó a partir del mes de febrero a noviembre del 2019 con su cronograma respectivo.

Se tomaron al azar a 376 patos adultos de Muscovy criados extensivamente en tres comunidades locales diferentes, que tienen características culturales y geográficas diferentes, en Guatemala: Camotán (Chiquimula), San Manuel Chaparrón (Jalapa) y Olintepeque (Quetzaltenango). Debido a limitaciones logísticas, no todas las mediciones fueron posibles en todas las aves, y aquellas con una o más mediciones que faltaron fueron excluidas del estudio. La muestra final fue de 362 patos adultos de Muscovy (137 machos y 225 hembras). Las mediciones se restringieron a aves aparentemente sanas que se ajustaban a los descriptores de clasificación de las especies. El manejo de animales se practicaba de acuerdo con la ética.
Los parámetros del estudio fueron los siguientes, de acuerdo con la literatura estándar (Cuesta, 2008), (Yakubu, 2011): Longitud del ala (húmero + cúbito + falanges) (LA); Longitud del miembro posterior (fémur + tibia + peroné + falanges) (LMP); Altura dorsal (AD); Longitud de la cabeza (LCz) y ancho de la cabeza (ACz); Longitud corporal (LC); Diámetro bicostal o ancho del cuerpo (DB); Diámetro longitudinal (DL); Diámetro dorsoesternal (DD); Altura de grupa (AG); Perímetro torácico (PT); Perímetro abdominal (PA); Perímetro del metatarso (PMt); Peso corporal (P).

Cuatro índices morfológicos se obtuvieron el corporal, compacidad, proporcionalidad y profundidad (Yakubu, 2011). El tipo de conformación y la musculatura de los patos se evaluó utilizando el índice de compacidad, corporal y proporcionalidad. Estos índices de selección importantes establecieron la proporción de mediciones que caracteriza la proporcionalidad del cuerpo del ave. La compacidad y la profundidad, que se utilizan para evaluar el desarrollo de la musculatura, son rasgos de solidez del cuerpo y rasgos claramente definidos para los patos tipo carne (Yakubu, 2011).

Para evaluar las diferencias entre sexos y localidades, se empleó un análisis de varianza multivariado no paramétrico de dos vías (NPMANOVA) con el sexo y la localidad como factores y rasgos, excluyendo índices, como variables dependientes, utilizando la distancia de correlación. Un MANOVA evaluó el dimorfismo sexual. Los NPMANOVA unidireccionales ulteriores y los valores corregidos de p-Bonferroni se aplicaron a la diferenciación entre comunidades para cada género por separado. Se obtuvo una tabla de correlación con las $r$ de Spearman. La agrupación jerárquica entre las variables morfométricas se evaluó según el método de Ward. Lue- 
go, se realizó un Análisis de Componentes Principales (ACP) de la matriz varcovar.

El análisis se realizó con el paquete PAST V. 2.17c (Hammer, Harper, \& Ryan 2001). Para múltiples pruebas estadísticas, se utilizó el ajuste secuencial de Bonferroni y 9.999 permutaciones, con un nivel de significación del $95 \%$.

\section{Resultados}

Un NPMANOVA bidireccional preliminar reflejó diferencias estadísticas entre las comunidades locales y los géneros (Tabla 1), por lo que procedemos a un análisis posterior con los sexos por separado. Las medias, las desviaciones estándar y los coeficientes de variación de los patos de los parámetros del cuerpo lineal se presentan en la tabla 2. La importancia con la minimización de la lambda de Wilks corroboró la diferencia entre géneros (Wilk's $\lambda=.254, \mathrm{~F} 14,347=72.8, \mathrm{p}<<.001$ ) y proporcionó validez para el dimorfismo sexual. Para las hembras no aparecieron diferencias estadísticas entre los animales entre San Manuel Chaparrón y Olintepeque $(F=$ $3.919, p=.109$ ), pero sí aparecieron para las hembras de Camotán. Para los machos, no aparecieron diferencias por comunidad.

La mayoría de las correlaciones entre las variables evaluadas fueron significativas ( $p$ $<.001$ ) y variaron de $2.8 \%$ a $73.4 \%$ (Tabla 3 ). La presencia de correlaciones positivas entre las variables evaluadas justifica el uso del análisis multivariado. El análisis grupal (Figura 1) demostró relaciones entre las variables morfométricas realizadas en dos grupos distintos. Un grupo estaba formado por variables que definen la longitud y el perímetro del animal (LC, LA, LMP, PA y PT) que se correlacionaron significativamente. Otro grupo comprendió las variables restantes.
Dos factores explicaron el $100 \%$ de la varianza total de las variables morfométricas estudiadas (PC1 + PC2 = 90.2 + 9.7\%). Los puntos en común encontrados en este estudio variaron de .617 a -.015 y esto explico cuánto contribuye una característica particular para decir el número de factores que se consideran (Figura 2). La característica de ACz mostró baja similitud, es decir, contribuyó poco a la varianza total observada. PA mostró la mayor similitud. PT fue la segunda variable más importante en el primer componente principal.

El primer factor puede denominarse "factor de perímetro" y las características de carga eran variables y altamente correlacionadas. Pero estas características no parecen ser importantes para describir una raza, sino más bien el manejo. LMP, LC y AD como variables más contribuyentes.

El rasgo muscular se describió mejor en los machos usando la compacidad (5.14 versus $4.09 \% ; p<.001$ para machos y hembras, respectivamente), mientras que, en las hembras, se explicó mejor a través de la profundidad (84.55 versus $8.15 \%$; $p \ll .001$ para hembras y machos respectivamente). La proporcionalidad fue más alta en las hembras $(84.43 \%)$ en comparación con los machos $(82.60 \%)(p=.012)$. Se encontró que el índice de corporal era más alto en machos $(6.08 \%)$ en comparación con las hembras $(4.92 \%)$ ( $p$ $<<.001)$. Se registraron coeficientes de variación más altos para los machos que para las hembras en todos los índices morfológicos, excepto para la relación corporal. 


\begin{tabular}{lcclcc}
\hline Variable & Suma de cuadrados & Grados de libertad & Cuadrado medio & $\mathbf{F}$ & $\mathbf{p}$ \\
\hline Municipio & 0.00747 & 2 & 0.0037377 & 12.612 & .0001 \\
Sexo & 0.00590 & 1 & 0.0059076 & 19.934 & .0001 \\
Interacción & -0.02524 & 2 & -0.0126240 & -42.599 & .4894 \\
Residual & 0.10550 & 356 & 0.0002963 & & \\
Total & 0.09363 & 361 & & & \\
\hline
\end{tabular}

Tabla 1. Resultados de NPANOVA de 2 vías con el sexo y el municipio como factores y rasgos como variables dependientes, utilizando la distancia de correlación y 9,999 permutaciones, en una muestra de 362 patos adultos de Muscovy (137 machos y 225 hembras) pertenecientes a tres municipios en Guatemala. Se reflejan diferencias estadísticas entre los municipios y los géneros.

\begin{tabular}{|c|c|c|c|c|c|c|c|c|c|c|}
\hline & \multicolumn{5}{|l|}{ q } & \multicolumn{5}{|l|}{$0^{\pi}$} \\
\hline & Min & Max & Media & Ds & $\mathrm{CV}$ & Min & Max & Media & Ds & $\mathrm{CV}$ \\
\hline LA & 3.5 & 42.0 & 31.5 & $(2.89)$ & 9.2 & 25.5 & 44.0 & 37.7 & (3.21) & 8.5 \\
\hline LMP & 22.0 & 38.0 & 3.9 & $(2.19)$ & 7.1 & 21.9 & 44.8 & 36.8 & (3.38) & 9.2 \\
\hline $\mathrm{LCz}$ & 1.0 & 14.5 & 12.7 & $(0.66)$ & 5.2 & 1.0 & 17.0 & 14.3 & $(0.95)$ & 6.6 \\
\hline LC & 31.0 & 49.0 & 36.7 & $(2.65)$ & 7.2 & 34.0 & 54.0 & 44.7 & (3.69) & 8.2 \\
\hline DB & 3.4 & 8.3 & 6.2 & $(0.72)$ & 11.7 & 4.2 & 9.3 & 6.9 & $(0.92)$ & 13.4 \\
\hline DD & 5.0 & 11.0 & 8.2 & $(0.99)$ & 12.0 & 5.5 & 11.7 & 9.2 & (1.19) & 12.9 \\
\hline DL & 11.0 & 19.0 & 14.3 & $(1.18)$ & 8.2 & 12.0 & 21.0 & 16.8 & (1.56) & 9.3 \\
\hline AG & 9.0 & 21.0 & 14.7 & (1.49) & 1.1 & 11.0 & 22.0 & 17.0 & $(1.87)$ & 11.0 \\
\hline PT & 25.5 & 37.0 & 3.9 & $(2.31)$ & 7.5 & 26.0 & 44.5 & 35.7 & $(3.58)$ & 1.0 \\
\hline PA & 18.0 & 38.0 & 25.8 & $(3.02)$ & 11.7 & 18.0 & 47.0 & 28.2 & $(4.06)$ & 14.4 \\
\hline PMt & 3.2 & 5.0 & 4.0 & $(0.34)$ & 8.5 & 3.5 & 7.0 & 4.8 & $(0.56)$ & 11.7 \\
\hline $\mathrm{ACz}$ & 2.4 & 4.0 & 3.4 & $(0.20)$ & 5.9 & 3.0 & 4.6 & 3.9 & $(0.30)$ & 7.6 \\
\hline $\mathrm{AD}$ & 13.0 & 24.0 & 17.4 & $(1.67)$ & 9.6 & 16.0 & 26.0 & 19.9 & $(2.09)$ & 1.5 \\
\hline BW & 1.0 & 2.4 & 1.5 & $(0.24)$ & 15.9 & 1.1 & 3.7 & 2.3 & $(0.54)$ & 23.6 \\
\hline
\end{tabular}

Tabla 2. Principales estadísticas simples para hembras $(n=225)$ y machos $(n=137)$ de patos Muscovy. Mediciones en $\mathrm{cm}$, excepto peso (en kg). Acrónimos en el texto. 


\begin{tabular}{lllllllllllllll}
\hline & LA & LMP & LCz & LC & DB & DD & DL & AG & PT & PA & PMt & ACz & AD & P \\
\hline LA & & .000 & .000 & .000 & .075 & .000 & .000 & .015 & .000 & .001 & .000 & .000 & .014 & .000 \\
LMP & .421 & & .000 & .000 & .000 & .000 & .000 & .007 & .000 & .000 & .000 & .000 & .002 & .000 \\
LCz & .264 & .381 & & .000 & .104 & .000 & .000 & .000 & .000 & .003 & .042 & .000 & .008 & .000 \\
LC & .364 & .506 & .334 & & .018 & .000 & .000 & .000 & .000 & .000 & .000 & .000 & .000 & .000 \\
DB & .119 & .347 & .109 & .157 & & .066 & .069 & .937 & .000 & .001 & .001 & .142 & .122 & .000 \\
DD & .232 & .440 & .301 & .413 & .123 & & .005 & .000 & .000 & .002 & .274 & .001 & .001 & .000 \\
DL & .289 & .383 & .256 & .283 & .121 & .185 & & .016 & .000 & .000 & .000 & .001 & .025 & .000 \\
AG & .162 & .178 & .234 & .243 & -.005 & .319 & .160 & & .038 & .673 & .017 & .013 & .000 & .001 \\
PT & .259 & .483 & .299 & .360 & .391 & .301 & .295 & .139 & & .000 & .000 & .001 & .000 & .000 \\
PA & .229 & .406 & .196 & .296 & .226 & .208 & .271 & -.028 & .586 & & .000 & .027 & .108 & .000 \\
PMt & .289 & .325 & .136 & .245 & .230 & .073 & .249 & .159 & .431 & .258 & & .000 & .000 & .000 \\
ACz & .348 & .343 & .364 & .253 & .098 & .214 & .217 & .166 & .217 & .148 & .248 & & .091 & .000 \\
AD & .163 & .209 & .175 & .314 & .103 & .212 & .150 & .734 & .249 & .107 & .269 & .113 & .384 & .246 \\
P & .404 & .500 & .273 & .310 & .345 & .336 & .280 & .213 & .725 & .535 & .396 & .000 \\
\hline
\end{tabular}

Tabla 3. Las correlaciones de Spearman entre las variables morfométricas evaluadas. Valores rs debajo de la fila diagonal; valores $p$ arriba. Acrónimos en el texto.

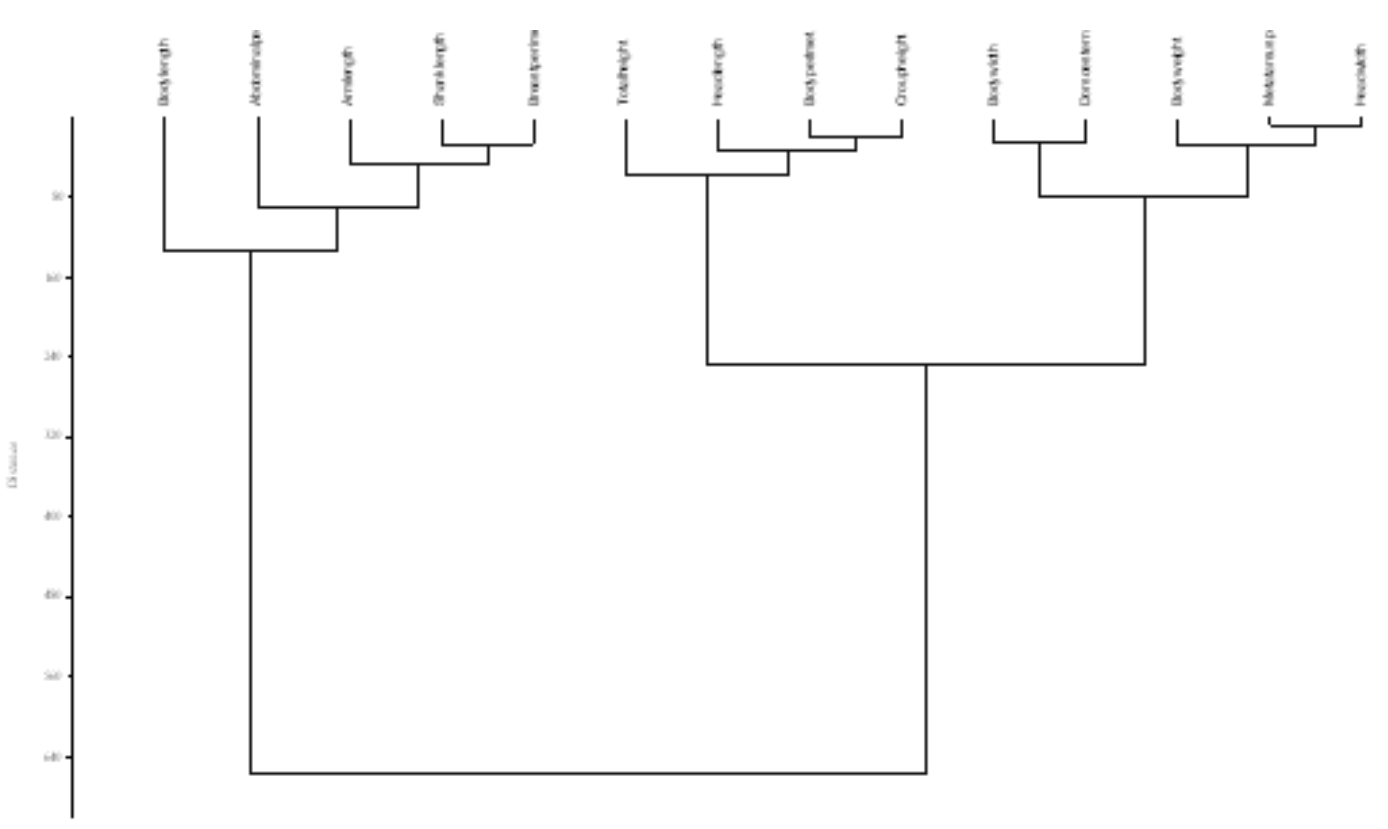

Figura 1. Análisis de variables por el algoritmo de Ward. Demuestra relaciones entre las variables morfométricas realizadas en dos grupos distintos. 


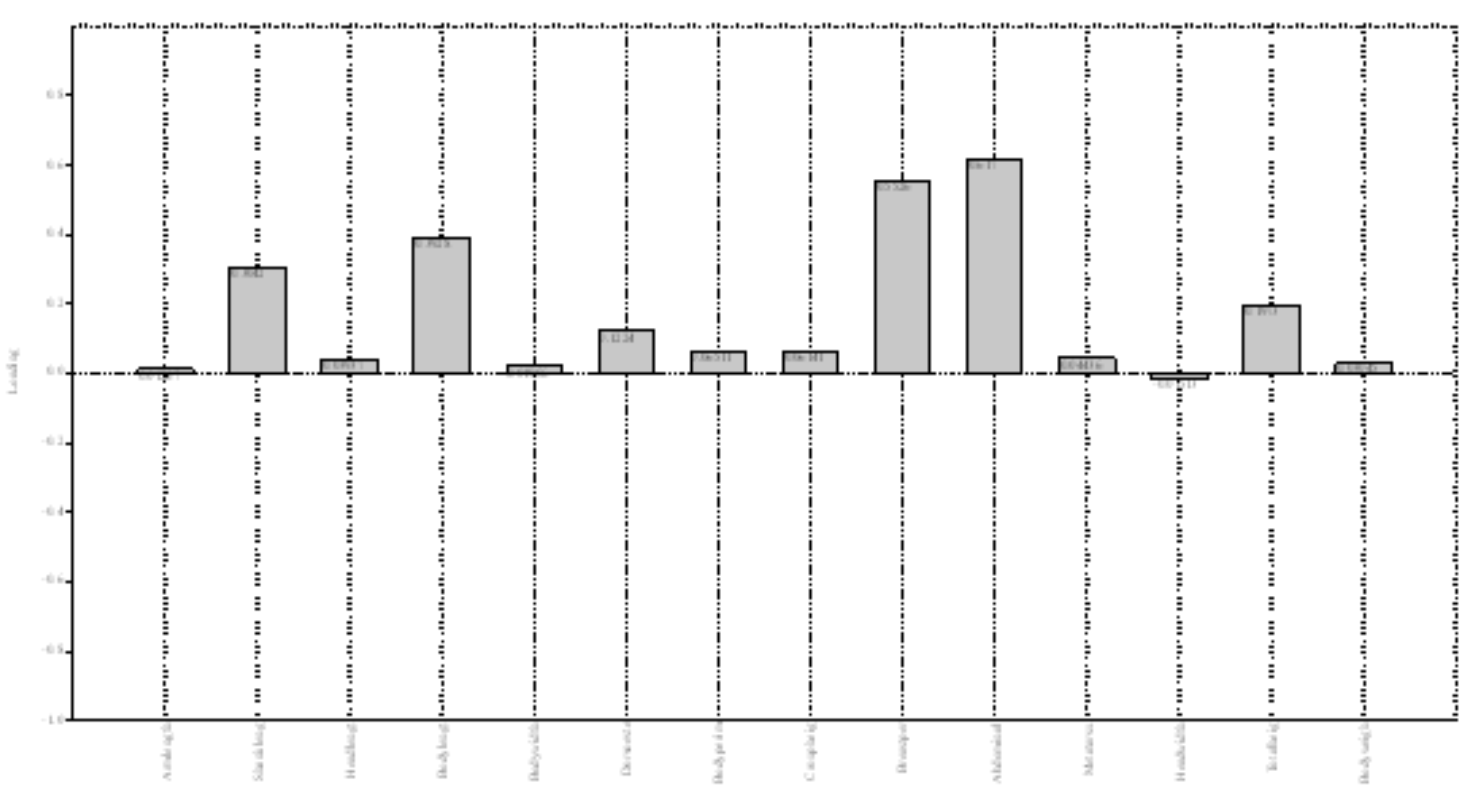

Figura 2. Cargas para análisis de componentes principales. Las comunidades de este estudio variaron de 0.617 a -0.015 y explicaron cuánto contribuye una característica en particular para decir el número de factores que se están considerando. Como todos los valores de carga fueron positivos, las diferencias sexuales se pueden considerar debido al tamaño.

\section{Discusión}

El pato es una de las especies de aves domésticas más importantes del mundo (Abdeltawab, et al., 2017). Los patos de traspatio Muscovy en Guatemala están bien adaptados a las condiciones climáticas locales, la alimentación y el estrés de manejo, con una mejor resistencia a las enfermedades de los trópicos, principalmente como resultado del estrés por calor frecuente y la sequía. Su variación fenotípica se debe probablemente a un simple manejo diferente, esto se expresa por la correlación del origen geográfico (comunidad) con algunos rasgos, como peso, pero los patos de los diferentes municipios no son distintos en función de las características morfológicas.

En los patos Muscovy, el dimorfismo sexual está bien manifestado. La alzada corporal más pequeña exhibida por las hembras en comparación con los machos puede ser un reflejo de su estrategia de adaptación a las condiciones ambientales y nutricionales estresantes, ya que un tamaño corporal más pequeño reduce los requerimientos de alimentación para el mantenimiento y aumentaría la eficiencia nutricional en este sistema extensivo donde la alimentación es limitada en términos de cantidad y calidad. En general, pareciera que hay una baja variación de rasgos fenotípicos que puede indicar una baja variación genética que podría perjudicar una respuesta en la selección. La alta variabilidad de peso en ambos sexos es una indicación de la alta sensibilidad ambiental de este rasgo de producción primaria.

El hecho de que se registraron coeficientes de variación más bajos para los machos para la relación de condición podría explicarse por la mayor ganancia promedio de peso corporal y un mayor crecimiento rela- 
tivo en los machos (Ismoyowati, Tugiyanti, Mufti, \& Purwantini, 2017), por lo que diferentes regímenes de manejo explicarían las divergencias en esa condición corporal.

La proporción más alta de pierna-cuerpo de las hembras es una indicación de que tienen piernas relativamente más largas, mientras que los machos tienen un cuerpo relativamente más largo. Este índice podría desempeñar un papel en la evaluación del tipo y la función. Mientras que las hembras muestran un cuerpo más estrecho, que es adecuado para la producción de huevos; los machos exhiben una apariencia más grande, que es más una característica presencia de músculo. Además, determinar la condición corporal es una herramienta valiosa, ya que puede usarse para indicar cómo los animales manejan la variación ambiental y los estresores (Angel, Wells, Rodríguez-Malagón, Tew, Speakman, \& Arnould, 2015). Entonces, si el peso se corrige para el tamaño del cuerpo usando la relación de condición, da una mejor indicación de la capacidad de un ave para cumplir con sus requisitos de energía presentes y futuros que usar solo el peso (Yakubu, Kaankuka. \& Ugbo, 2011). El índice de condición más alto detectado en los hombres es de importancia fisiológica porque las medidas estándar de las actividades metabólicas se expresan con frecuencia en función del tamaño del cuerpo, y a menudo es útil examinar la relación de las estructuras u órganos en relación con el tamaño corporal total (Yakubu, et al., 2011).

Con respecto a los datos obtenidos, los patos nativos de Muscovy tienen rasgos morfológicos y biométricos que podrían representar genes de adaptación a su propio entorno. Los patos de traspatio deben verse como reservorios genéticos que reflejen una adaptación única a sus ambientes agroecológicos o tropicales.
Los patos criollos de Muscovy en Guatemala se crían en diferentes matrices ecológicas y, por lo tanto, la diferenciación podría notarse en diferentes regiones y altitudes. Los rasgos morfológicos entre las hembras también indican un posible grupo diferente en Camotán, que podría ser un prototipo, pero es un grupo asociado simplemente con la selección artificial y la cría, no con la selección natural. Ahora sería deseable registrar los rendimientos de crecimiento bajo el sistema libre y analizarlo.

El estudio de caracteres fenotípicos es la base para la diferenciación de grupos y / o razas y proporciona apoyo para programas de conservación; Es una técnica utilizada para caracterizar los recursos genéticos en muchos países. Hay muchas herramientas disponibles que pueden ayudar a determinar el poder discriminatorio que tienen las variables al describir los patrones de raza.

Se ha utilizado ampliamente en estudios de caracterización de razas y diversidad genética, ya que proporciona un análisis descriptivo de las diferencias entre las poblaciones, considerando todas las variables juntas, proporcionando una visión general de los datos. El análisis multivariado permite un mayor poder de discriminación, eliminando las variables difíciles de medir y las que contribuyen poco a explicar la variación. Por lo tanto, el objetivo de este estudio fue reducir la dimensionalidad de los datos, eliminar la información redundante e identificar similitudes mediante el uso de análisis multivariado.

El estudio reveló que había marcadas diferencias sexuales en las mediciones morfológicas de los patos de Muscovy con valores más altos en la mayoría de los casos registrados para los machos. Ahora es necesario estudiar la calidad de la canal y del huevo y otras variaciones a niveles moleculares que aclararán 
aún más la similitud con los patos de otros orígenes para registrar y registrar esta raza internacionalmente. Estos germoplasmas de pato de Guatemala son conocidos por su producción bajo un sistema de manejo de insumos nulos, otros estudios que se centren en la caracterización de estos patos a nivel molecular serán el mejor enfoque para la selección y conservación adecuadas de este germoplasma único para uso y selección futuros.

\section{Agradecimientos}

La ejecución y redacción del presente estudio no hubiera sido posible sin el cofinanciamiento de la Dirección General de Investigación de la Universidad de San Carlos de Guatemala, programa de recursos naturales y ambiente, partida presupuestal 4.8.24.1.04, 2019. La participación del Licenciado Zootecnista Luis Vásquez Chegüen, estadístico del proyecto y en especial a las carreras de Zootecnia del Centro Universitario de Oriente y del Centro Universitario del Sur Oriente de la Universidad de San Carlos de Guatemala, así también, a todas las familias que aportaron su conocimiento y por el apoyo para llevar a la culminación este proyecto.

\section{Referencias}

Abdeltawab, M.A. Salha, E.A. Allam, H.Z. \& Fandy, W. A. Karyological (2017) Studies on Some Breeds of Duck, Minia Journal Agriculture Res. Dev., 37(1), 61-81.

Almeida et al., (2014) Dimorfismo Sexual Do Pato Doméstico (Cairina moschata) Utilizando Análise Multivariada, Actas Iberoamericanas de conservación animal., 4, 53-55.

Angel, L., Wells, M.R. Rodríguez-Malagón, R.A., Tew, E. Speakman, J.R., \& Arnould,
J.P. (2015). Sexual size dimorphism and body condition in the Australasian Gannet, PLoS One, 10(12) 1-16.

Baéza, E., Williams, J., Guémené, D., \& Duclos, M. J. (2001). Sexual dimorphism for growth in Muscovy ducks and changes in insulin-like growth factor I (IGF-I), growth hormone $(\mathrm{GH})$ and triiodothyronine (T3) plasma levels. Reproduction Nutrition Development, 41(2), 173-179.

Cuesta, M. L. (2008) Pictorial guidance for phenotypic characterization of chickens and ducks, Rome.

Donkin, R.A. (1989) The Muscovy duck, Cairina moschata domestica: origins, dispersal, and associated aspects of the geography of domestication. Rotterdam: Brookfield, VT.

Etuk, L.F. Abasiekong, S.F. Ojewola, G. S. \& Akomas, S.C. (2006) Carcass and organ characteristics of muscovy ducks reared under three management systems in South Eastern Nigeria, International Journal Poultry Science. 5(6), 534-537.

FAO, (2006) Animal genetic resource conservation by management, databanks and training, Rome.

Fitsum, M. (2015) Phenotypic characterization of local chicken ecotype in the Central zone of Tigray in Northern Etiopia, Jimma University, Ethiopia.

Hammer, O. Harper, D.A. \& Ryan, P.D. (2001) Paleontological statistics software package for education and data analysis $v$. 2.17c, Palaeontol. Electron, 4(1), 1-229.

Hernández, D. Muñoz, N. Valencia, Posso, A. \& Muñoz, J.E. (2007) Caracterización molecular del pato criollo colombiano en cuatro departamentos, Acta Agronómica, 56, (3), 141-145. 
Igwe, G. Okoli, I. \& Okeudo, N. (2003) Hematological Characteristics of Ducks (Cairina moschata) of Southeastern Nigeria, Tropicultura, 21(2), 61-65.

Ismoyowati, E. Tugiyanti, M. Mufti, M. \& Purwantini, D. (2017) Sexual dimorphism and identification of single nucleotide polymorphism of growth hormone gene in muscovy duck, Journal Indonesia Tropical. Animal Agricola, 42(3), 167-174.

Jáuregui, R. Flores, H. Vásquez, L. \& Oliva, M.J. (2015) Caracterización morfométrica de la gallina de cuello desnudo (Gallus domesticus nudicollis) en la región ch'ortí de Chiquimula, Guatemala, Ciencia, Tecnología y Salud, (2) 1-8.

Ogah, D.O. (2011) Assessing Size and Conformation of the Body of Nigerian Indigenous Turkey," Slovak. Journal Animal Science 44(1), 21-27.

Oguntunji A.O. \& Ayorinde, K.L. (2014) Multivariate analysis of morphological traits of the Nigerian Muscovy ducks (Cairina moschata), Archivos Zootecnia., 63(243) 483-493.
Parés-Casanova, P.M. (2013) Morphological similarities between spanish pigeon breeds, Turkish Journal Veterinary Animal Science 37(3),346-351.

Schaaf, A., Gomez, D. Tallei, E, Rivera, O. Politi, N. \& Cuyckens, G. A. (2018) Assessing distribution and conservation potential for the muscovy duck (Cairina moschata) in Argentina, Neotropropical Biology Conservy 13(3), 183-191.

Uribe, F. Senar, J.C. \& Camerino M. (1985) Morfometría de las palomas semidomésticas (Columba livia var.) de la Ciudad de Barcelona, Arx. Miscellànea Zoològica, 9, 339-345.

Yakubu, A. (2011) Discriminant analysis of sexual dimorphism in morphological traits of African Muscovy ducks, Archivos Zootecnia., 60(232),1115-1123. Yakubu, A. Kaankuka,F. \& Ugbo, S. (2011) Morphometric Traits of Muscovy Ducks from Two Agro Ecological Zones of Nigeria, Tropicultura, 29(2), 121-124.

\section{Sobre autor}

\section{Dr. Raúl Jáuregui Jiménez}

Médico Veterinario egresado de la Facultad de Veterinaria y Zootecnia de la USAC, tiene una Maestría en Salud Animal egresado de la Universidad Autónoma del Estado de México, Toluca México. En la docencia he trabajado en la carrera de zootecnia desde sus inicios cuando se desarrollo el programa de técnicos universitarios, posteriormente en 1993 se inició el programa de licenciatura en zootecnia en donde participé en las adecuaciones curriculares de la carrera y coordine dicha carrera durante diez años. A partir de 1998 inicie la fase de investigador en donde al inicio desarrolle trabajos sobre epidemiología veterinaria y sanidad animal, posteriormente funde la linea de investigación sobre la conservación y utilización de los recursos zoogenéticos del país enfocado a los animales domésticos de traspatio del área rural. paralelamente se trabajó otra línea de investigación sobre la inocuidad de los alimentos de origen animal específicamente la leche de bovino. 


\section{Sobre coautores}

\section{Mtro. Mario Efraín González Estrada}

Técnico en Producción Pecuaria y Técnico en Producción Agrícola egresado del Centro Universitario de Sur Oriente CUNSURORI, Licenciado en Zootecnia del Centro Universitario de Sur Oriente CUNSURORI. Posee una Maestría en Producción Avícola egresado de la Facultad de Medicina Veterinaria y Zootecnia de la Universidad de San Carlos de Guatemala. Tiene un posgrado en Biotecnologías Reproductivas en Pequeños Rumiantes de la Universidad Autónoma de Chiapas, Campus IV, Huehuetan (2010). Posgrado de Actualización en la Introducción a la Alimentación Animal en el Departamento de Estudios de Posgrado de la Facultad de Medicina Veterinaria y Zootecnia, USAC (2019). Seminario de Investigación Cuantitativa en la Universidad de Costa Rica, UCR. (2019). Es Profesor Investigador Titular $\checkmark$ de la Carrera de Zootecnia del Centro Universitario de Sur Oriente CUNSURORI. Coordinador de la Carrera de Zootecnia del CUNSURORI. Miembro de la Comisión de Investigación del Centro Universitario de Sur Oriente, USAC. Secretario de la Comisión de Recursos Zoogeneticos del Colegio de Médicos Veterinarios y Zootecnistas de Guatemala. Investigador con registro en la Dirección General de Investigación DIGI de la Universidad de San Carlos de Guatemala y de la Secretaria Nacional de Ciencia y Tecnología SENACYT, Guatemala.

\section{Lic. Carlos Roberto Lorenzo Machorro}

Técnico en Producción Pecuaria egresado del Centro Universitario de Oriente -CUNORI- , USAC, Licenciado Zootecnista egresado del Centro Universitario de Oriente -CUNORI- , USAC. Laborando como Investigador del Centro Universitario de Oriente y de La Dirección General de Investigación de la Universidad de San Carlos de Guatemala. Experiencia en Investigación relacionada a la caracterización para la conservación de especies nativas de traspatio como la Caracterización del cerdo criollo en la Región Chorti en el Departamento de Chiquimula y de igual forma con el pato criollo para los Departamentos de Chiquimula, Jalapa y Quetzaltenango.

\section{Dra. Anna Isern Sabria}

La Doctora Anna Isern Sabria es originaria de Catalunya, España. Es Doctora en Veterinaria Rural por la Universidad Autónoma de Barcelona, y ha realizado una Maestría en Cooperación al Desarrollo y un Diplomado en Género e Interculturalidad. Llegó a Guatemala en el año 1993 como cooperante de la ONG Veterinarios Sin Fronteras, y durante 20 años fue la Coordinadora Regional de Centroamérica de Veterinarios Sin Fronteras -España-

En la actualidad es Consultora Internacional en el tema de Etnoveterinaria y realiza trabajos de investigación para el rescate de la Etnoveterinaria en comunidades indígenas de Guatemala en colaboración con la REDSAG (Red de Soberanía Alimentaria en Guatemala). 
Entre sus publicaciones están:

-Libros: Etnoveterinaria en Guatemala y sus orígenes, 2004 y Escuela de Campo de Agricultores en Producción pecuaria sostenible, 2007

-Manuales: Colección de cuatro Manuales de Etnoveterinaria para la formación de promotores etnoveterinarios en Guatemala, 2,003. Manual de Etnoveterinaria Q'eqchí 2015 y Manual de Etnoveterinaria K'iché 2019.

\section{Dr. Pere-Miquel Parés Casanova}

El Doctor Pere-Miquel Parés Casanova es originario de Catalunya, España. Es Ph D en Veterinaria Rural por la Universidad Autónoma de Barcelona. Actualmente es catedrático del Departamento de Ciencia Animal, de la Escuela Técnica Superior de Ingeniería Agraria en la Universidad de Lérida, Catalunya, España. Colabora con la Red CONBIAND en Guatemala desde el año 2014 con diversos estudios de investigación Morfométrica sobre las Ovejas Criollas y las Aves Criollas de Traspatio.

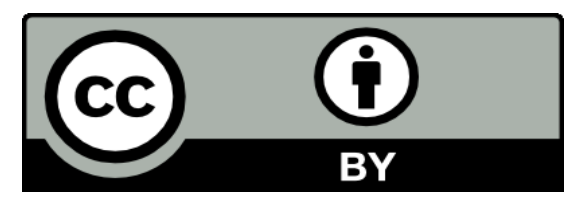

Este texto está protegido por una licencia CreativeCommons 4.0.

Esta licencia permite que otros distribuyan, mezclen, adapten y desarrollen su trabajo, incluso comercialmente, siempre y cuando le den crédito por la creación original. 